\title{
Microstructural White Matter Alterations in Cognitively Impaired Patients at Early Stages of Multiple Sclerosis
}

\author{
Ruth Schneider ${ }^{1,2} \mathbb{D}$ - Britta Matusche ${ }^{2} \cdot$ Erhan Genç $^{3} \cdot$ Ralf Gold $^{1} \cdot$ Barbara Bellenberg $^{2} \cdot$ Carsten Lukas $^{2,4}$
}

Received: 25 August 2020 / Accepted: 26 February 2021 / Published online: 31 March 2021

(c) The Author(s) 2021

\begin{abstract}
Purpose As conventional quantitative magnetic resonance imaging (MRI) parameters are weakly associated with cognitive impairment (CI) in early multiple sclerosis (MS), we explored microstructural white matter alterations in early MS or clinically isolated syndrome (CIS) comparing patients with or without CI.

Methods Based on a preceding tract-based spatial statistics analysis (3 Tesla MRI) which contrasted 106 patients with early MS or CIS and 49 healthy controls, diffusion metrics (fractional anisotropy, FA, mean diffusivity, MD) were extracted from significant clusters using an atlas-based approach. The FA and MD were compared between patients with $(\mathrm{Ci} P \mathrm{P} n=14)$ and without (Cp_P $n=81$ ) cognitive impairment in a subset of patients who underwent CI screening.

Results The FA was reduced in $\mathrm{Ci} \_\mathrm{P}$ compared to $\mathrm{Cp}$ _P in the splenium of corpus callosum $(p=0.001)$, right parahippocampal cingulum $(p=0.002)$ and fornix cres./stria terminalis $(0.042)$, left posterior corona radiata $(p=0.012)$, bilateral cerebral peduncles, medial lemniscus and in cerebellar tracts. Increased MD was detected in the splenium of corpus callosum $(p=0.01)$. The CI-related localizations overlapped only partially with MS lesions.

Conclusion Microstructural white matter alterations at disease onset were detectable in Ci_P compared to Cp_P in known cognitively relevant fiber tracts, indicating the relevance of early treatment initiation in MS and CIS.
\end{abstract}

Keywords Clinically isolated syndrome $\cdot$ Cognitive impairment $\cdot$ Fractional anisotropy $\cdot$ Mean diffusivity $\cdot$ Tract based spatial statistic

Availability of Data and Material (data transparency) The datasets for this manuscript are not publicly available for reasons of patient confidentiality. Requests to access the datasets should be directed to the corresponding author RS (ruth.schneider@rub.de).

Code Availability (software application or custom code) All software applications used for analyses of this study (except for statistics) are freely available (public domain).

Ruth Schneider

Ruth.Schneider@rub.de

1 Department of Neurology, St. Josef Hospital, Ruhr-University Bochum, Bochum, Germany

2 Institute of Neuroradiology, St. Josef Hospital, Ruhr-University Bochum, Bochum, Germany

3 Department of Psychology and Neurosciences, Leibniz Research Center for Working Environment and Human Factors, Dortmund, Germany

4 Department of Diagnostic and Interventional Radiology and Nuclear Medicine, St. Josef Hospital, Ruhr-University Bochum, Bochum, Germany

\section{Introduction}

Cognitive impairment (CI) is a frequent symptom with prevalence of $40-65 \%$ in multiple sclerosis (MS). It has been attributed to damage of brain white matter (WM) as well as to brain grey matter (GM), both of which can be caused by demyelination, inflammation and axonal loss [1]. CI has already been reported in early disease stages of MS and clinically isolated syndrome (CIS) with a prevalence ranging from $13 \%$ to $20 \%$ [2-4]. Due to the fact that CI is highly relevant for occupational disability and restrictions in daily life, the early onset of CI is indicative of future quality of life for MS patients [5]. As a result, efforts have been made to identify risk factors to determine and predict CI based on clinical markers (Expanded disability status sclae (EDSS), disease duration) and conventional magnetic resonance imaging (MRI) parameters (brain lesion load and location in T1 and T2-weighted MRI, brain atrophy) [6, 7]; however, associations between conventional MRI parameters, such as brain atrophy or lesion load, and CI were weak, especially in young MS patients with short disease dura- 
tion [8]. The lack of association between conventional MRI characteristics and baseline CI was recently confirmed in a large multicenter cohort study on patients first diagnosed with MS or CIS [9].

Thus, there is a need for more sensitive MRI parameters to identify structural brain differences between patients with and without CI, particularly in the early stages of MS. In this context, microstructural tissue damage detectable by diffusion tensor imaging (DTI) seems to be a promising complementary marker in addition to grey matter atrophy [7, 10]. DTI facilitates microstructural WM quantification in normal-appearing WM as well as in MS-related lesions by diffusion metrics, such as fractional anisotropy (FA), mean, axial and radial diffusivity (MD, AD, RD). Both FA and MD are regarded as measures of disrupted fiber integrity and increased mobility of water molecules, while $\mathrm{RD}$ and $\mathrm{AD}$ have been suggested as markers of myelin and axonal damage, respectively $[11,12]$.

In order to spatially localize changes of these diffusion-related metrics, tract-based spatial statistics (TBSS) has been developed, which provides voxel-wise statistics on a whole brain skeletonized representation of the major WM fiber tracts [13]. A TBSS study involving longstanding MS demonstrated that patients with CI had more severely altered DTI metrics than cognitively preserved patients. These changes were partly found in regions that are considered specific for CI (i.e., the corpus callosum, CC, cingulum, posterior thalamic tract, superior and inferior longitudinal fasciculus, forceps major, cingulum, fornices) but also in the corticospinal tracts [13]. In patients with CIS or early MS WM tract alterations including corticospinal, interhemispheric and association fiber pathways investigated by TBSS have been reported [14]. In a recent TBSS study of our own group (including 106 patients at first diagnosis of MS or CIS) we detected widespread microstructural alterations compared to healthy controls (HC) in all major white matter pathways including callosal, association, limbic, corona radiata, corticospinal and cerebellar pathways. Herein, similar structures were involved in patients with CIS compared to patients with early MS [15].

On the basis of these previous findings the present study aimed at further characterizing the microstructural differences within these altered regions with respect to their relationship to CI. We therefore classified our patients into cognitively impaired (Ci_P) and cognitively preserved patients (Cp_P) and considered DTI metrics in those regions in which significant WM alterations have been found in our preceding study between patients and HC. We studied differences between patients who were assigned to $\mathrm{Ci}_{-} \mathrm{P}$ or Cp_P after first diagnosis of MS or CIS to identify relevant clusters in WM which might be related to CI. We hypothesized that even during early disease course of MS or CIS differences in WM integrity between $\mathrm{Ci}_{-} \mathrm{P}$ and $\mathrm{Cp} \_\mathrm{P}$ will be detected and that in turn microstructural WM damage on fibers related to cognitive processing will be associated to $\mathrm{CI}$.

\section{Methods}

\section{Patient and Healthy Controls}

A total of 106 patients (CIS, $n=51$ ) and early relapsing remitting MS (RRMS, $n=55$ ) after a first clinical episode of MS were enrolled in a single center taking part in an ongoing prospective longitudinal multicenter cohort study (NationMS) of the German Competence Network Multiple Sclerosis (KKNMS). According to the study design, the enrolled patients were treatment-naive to disease-modifying drugs at study entry and had either a diagnosis of CIS with a high risk of conversion to MS within 6 months, or early definite MS less than 24 months after onset of symptoms [16]. The patients were compared to an age and sex matched healthy control group (HC, $n=49$ ). The study was approved by the university local ethics committee (approval No. 3714-10) and all patients provided written informed consent prior to study participation.

A subset of 95 patients who received screening for CI were included in the following analyses of the present study. There were no significant differences regarding age, EDSS or brain lesion load between the groups of patients who did or did not receive cognitive screening. The results are shown in the Online_Resource_1.

\section{Cognitive Screening and Disability}

Screening for CI was performed using the Multiple Sclerosis Inventory for Cognition (MUSIC) [17]. MUSIC is a rapid (about 10-12 min) multiple domain cognitive screening test reflecting the most frequently impaired cognitive domains in MS (verbal fluency, verbal working memory and memory retrieval, visual-spatial processing speed and executive functions), which is widely used in German-speaking countries. A timed verbal fluency task with change of categories reflects verbal fluency and executive functions. Working memory functions and attention are assessed using immediate wordlist recall tasks and memory retrieval using a delayed word list recall tasks. A modified Stroop test (naming of animal silhouettes either in a congruent or incongruent condition) assesses information processing speed and executive functions. For each participant the raw scores are converted into a total sum score $($ maximum $=30$ ) reflecting general cognitive functioning on the basis of normative data of 158 healthy controls controlling for age, sex and education. The contributions of the subscores to the sum score are weighted 


\section{Preceding Study \\ (Schneider et al., Front. Neurol. 2019)}

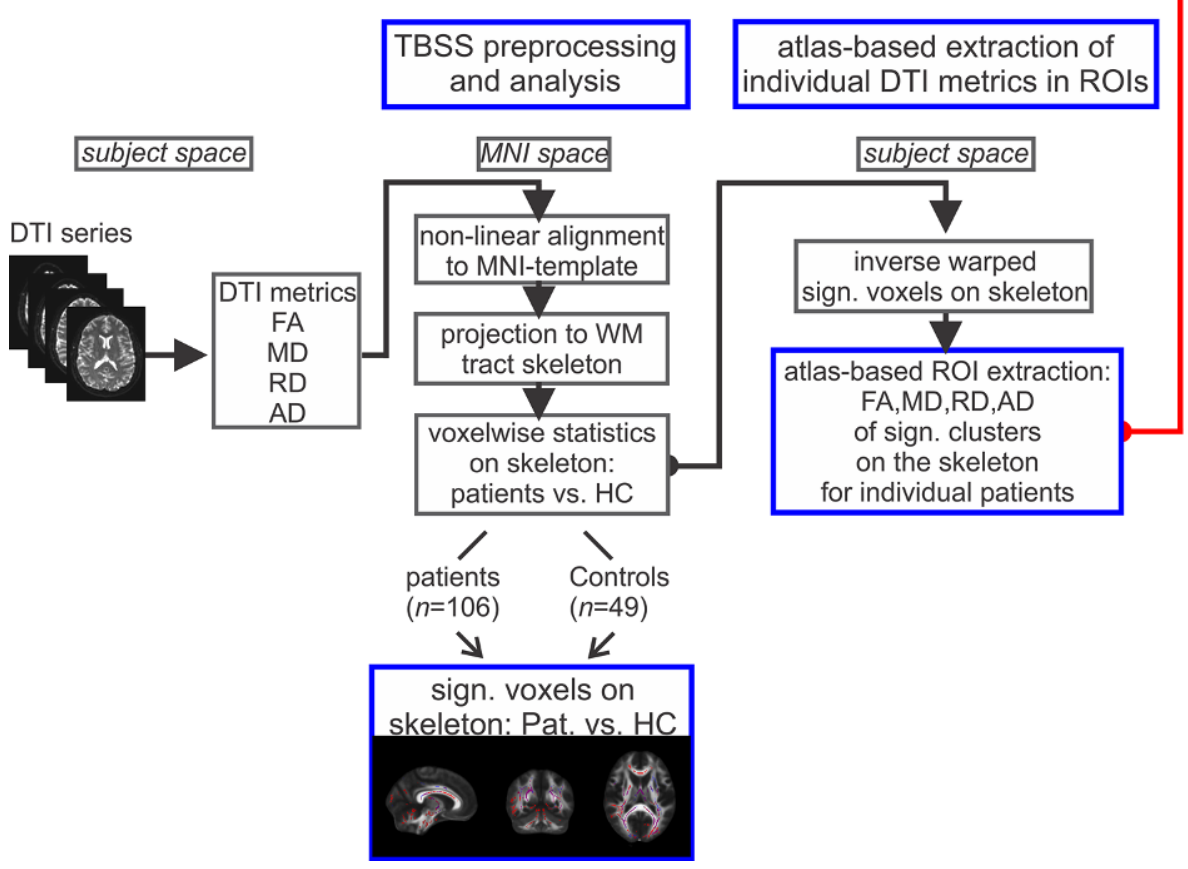

\section{Present Study}

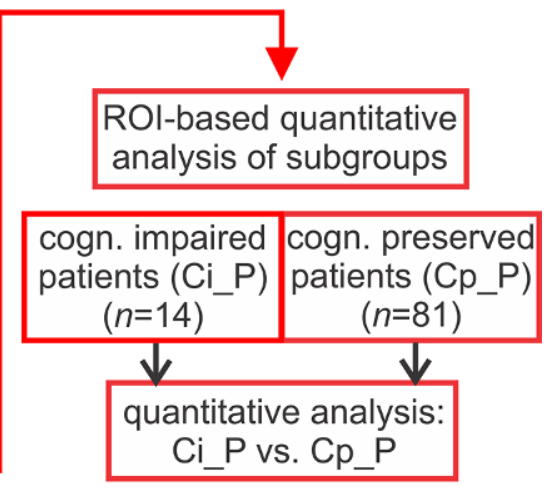

Fig. 1 Study design: built on atlas-based region of interest (ROI) extraction the present study investigated (red framed elements) quantitative diffusion tensor imaging (DTI) metrics of cognitively impaired patients (Ci_P, $n=14)$ in comparison to cognitively preserved patients $\left(\mathrm{Cp} \_\mathrm{P}\right.$, $n=81$ ). It was based on the results of a preceding tract-based spatial statistics (TBSS) analysis (blue framed elements) which yielded individual fractional anisotropy (FA), mean diffusivity (MD), radial diffusivity (RD) and axial diffusivity (AD) values in clusters on the white matter (WM) skeleton in which significant differences between patients and healthy controls (HC) have been detected [15]. MNI Montreal Neurological Institute, cogn. cognitive, Pat. patients, sign. significant, vs. versus (compared against)

on the basis of their respective diagnostic value (superior sensitivity and specificity receiving a higher weight), such that the sum score equally reflects impairment of working memory functions (word recall tasks) and higher executive functions (verbal fluency task and modified Stroop test) $[18,19]$. The MUSIC cognition score ranges from 0 to 30 , with values $\leq 10$ representing marked cognitive dysfunction, 11-15 representing medium grade cognitive dysfunction, 16-20 at least mild cognitive impairment, and $>20$ cognitive performance in the normal range. According to the MUSIC rating scale, patients with values higher or equal than 20 were defined as cognitively preserved (Cp_P) while patients with values below 20 were defined as at least mildly cognitive impaired (Ci_P) [20]. Additionally, all patients underwent neurological examinations including the expanded disability status scale (EDSS) [21].

\section{MR Imaging}

Imaging was performed using a single 3T scanner (Achieva Philips, Best, The Netherlands) with a standardized imaging protocol including a single-shot $2 \mathrm{D}$ echo planar imaging (EPI) sequence for diffusion-weighted imaging (50 axial slices, $2.5 \mathrm{~mm}$ slice thickness, field of view of $320 \times 240$, in- plane resolution of $2.5 \times 2.5 \mathrm{~mm}^{2}$, TR/TE $7000 / 90$, flip angle of $90^{\circ}, 32$ gradient directions with b-value $=900 \mathrm{~s} / \mathrm{mm}^{2}$ and one volume, two averages, without diffusion weighting, acquisition time of 5min 11s) and an isotropic 3D fluid attenuated inversion recovery (FLAIR) sequence for lesion quantification (170 sagittal slices, field of view of $240 \mathrm{~mm}$, resolution of $1 \times 1 \times 1 \mathrm{~mm}^{3}$, TR/TE/inversion time in $\mathrm{ms}$ of $4800 / 286 / 1650$, turbo factor of 182 , acquisition time of $6 \mathrm{~min} 30 \mathrm{~s})$. A structural isotropic T1-weighted 3D sequence (T1 fast field echo; 180 sagittal slices; FOV: $240 \mathrm{~mm} \times 240 \mathrm{~mm}$; voxel size: $1 \mathrm{~mm} \times 1 \mathrm{~mm} \times 1 \mathrm{~mm}$; TR, TE, TI/ms: 10/4.6/1000; flip angle: $8^{\circ}$, turbo factor: 164 ; acquisition time: $6^{\prime} 00^{\prime \prime} \mathrm{min}$ ) was also included.

\section{Quantitative Analyses}

For this study we used quantitative DTI metrics which were assessed based on a recent preceding study of our group [15]. In that study we conducted TBSS analyses including the participants described above to investigate differences in WM tract integrity between patients and HC. Therein, the detected FA and MD alterations in patients with CIS or RRMS compared to HC were widespread all over the brain and involved most supratentorial and infratentorial 
WM tracts. FA reductions also involved the cerebellum. $\mathrm{AD}$ was increased in patients compared to $\mathrm{HC}$ in the right superior corona radiata, while there were no significant $\mathrm{RD}$ differences between patients and HC.

We extracted individual quantitative results of the DTI metrics for each participant in all clusters in which significant alterations between patients and $\mathrm{HC}$ had been found. A summarizing figure showing the significant clusters of FA and MD alterations on the WM tract skeleton is provided as an online resource (Online_Resource_1). In the present study we analyzed these quantitative DTI results to compare Ci_P and Cp_P. Fig. 1 illustrates the entire study design incorporating the preceding analyses and the present study. In the following Sect. 2.4.1 and 2.4.2 we summarize the methodology which was used in the already published preceding study [15]. For further details of the TBSS analysis and atlas-based extraction of DTI metrics in significant clusters on the WM tract skeleton we refer to [15].

\section{TBSS Analysis of Patients and Healthy Controls (Summary of [15])}

In brief, we used tract-based spatial statistics (TBSS, version 1.2) as a part of the FSL software package [22, 23] with default settings as described in the TBSS manual to perform voxel-wise statistics of DTI data (https://fsl.fmrib.ox. ac.uk/fsl/fslwiki/TBSS/UserGuide; Fig. 1). First, FA, MD, $\mathrm{RD}$ and $\mathrm{AD}$ images were generated from the raw diffusion-weighted data. Second, FA images were warped to a $1 \times 1 \times 1 \mathrm{~mm}^{3}$ standard space target image (FMRIB58_FA) using nonlinear registration and a mean FA skeleton, which represented the centers of all tracts common to the group was generated. The aligned FA, MD, RD and AD data were projected onto this skeleton and the resulting skeletonized data were used to perform voxel-wise statistics between patients and $\mathrm{HC}$.

Between-group analyses were conducted using permutation methods for non-parametric voxel-wise statistical analysis (FSL randomize tool) [24] (two-sample unpaired t-tests with age and sex as nuisance variables). We identified regions of the WM tracts showing significant group differences in the TBSS analyses (clusters) with significantly altered DTI parameters using threshold-free cluster enhancement at a significance threshold of $p<0.01$.

\section{Region of Interest (ROI) Based Extraction of Quantitative DTI Metrics (Summary of [15])}

For each participant we extracted mean values and standard deviations of the DTI metrics from the relevant clusters using the image statistics utilities in FSL (FSLUTILS: fslmaths, fslmeants, fslstats) by attributing them to regions of the DTI-based brain white matter label atlas (JHU-
ICBM-labels-1 mm) [25] and the probabilistic cerebellar atlas (Cerebellum-MNI_fnirt_1 mm, at a $25 \%$ threshold) [26]. As detailed in the online resource, the relevant clusters affected all regions of the JHU-label atlas and the main ROIs of the cerebellar atlas which were included in the further analyses.

\section{Cognition-related Group Analysis}

The resulting quantitative DTI results were further analyzed for the subset of patients who received cognitive testing to assess differences between $\mathrm{Ci}$ _P and Cp_P.

\section{Lesion Quantification}

We generated lesion probability maps for $\mathrm{Ci}_{-} \mathrm{P}$ and $\mathrm{Cp} \_\mathrm{P}$ subgroups to assess whether there was an overlap between lesions and relevant ROIs extracted from the TBSS analysis. Individual lesion probability maps and total FLAIR lesion volume were obtained for each patient using the lesion prediction algorithm in LST toolbox version 3.0.0 (www.statistical-modelling.de/lst.html) for SPM [27]. During the lesion segmentation procedure, FLAIR images and resulting lesion maps were coregistered to the corresponding 3D-T1-weighted series. The individual T1-weighted series were registered and normalized to the DARTEL template in MNI space using the preprocessing procedures of the CAT12 segmentation tools (CAT12, version R1165, http://www.neuro.uni-jena.de/hbm2016/GaserHBM2016.

pdf). Deformation fields of the T1-transformations were applied to the individual lesion probability maps to transform them to the common MNI-space using the SPM12 Normalize (write) function. We generated single, averaged lesion probability maps for both subgroups (Ci_P and Cp_P) using the SPM12 image calculator tool. For visual representation in the FSL image viewer FSLeyes, the lesion probability maps were thresholded at a level of 0.15 , showing regions where at least $15 \%$ of the patients had FLAIR lesions.

\section{Statistics}

The IBM SPSS software (version SPSS 24) was used for further statistical analyses of quantitative results. For all statistical analyses in SPSS, linear parametric or non-parametric methods were used, and testing was two-tailed with an $\alpha$-level of $p<0.05$. Comparisons of demographic data were assessed by one-way ANOVA (controlled for age and disease duration), Mann-Whitney U-tests (for EDSS and lesion load) or $\chi^{2}$-tests (for numbers of participants). The association between quantitative DTI parameters in the ROIs and performance in cognition was investigated using a general linear multivariate model including CI status and sex 
Table 1 Summary of demographic and clinical data; classification into cognitively impaired (Ci_P) or cognitively preserved (Cp_P) according to screening test (MUSIC cognition) for CI. The columns "All patients" and "healthy controls" refer to the participants of the preceding TBSS analysis [15]

\begin{tabular}{|c|c|c|c|c|c|}
\hline & Ci_P & $\mathrm{Cp} \_\mathrm{P}$ & All patients & $\mathrm{HC}$ & $p$-value \\
\hline$n$ & 14 & 81 & 106 & 49 & - \\
\hline Female/male & $6 / 8$ & $55 / 26$ & $68 / 38$ & $32 / 17$ & n.s. ${ }^{a}$ \\
\hline Age (years) mean \pm SD & $40 \pm 10$ & $36 \pm 11$ & $37 \pm 11$ & $42 \pm 14$ & $\begin{array}{l}\text { Ci_P vs. Cp_P: n. s. } \\
\text { All patients vs. HC: } p<0.05^{b}\end{array}$ \\
\hline $\begin{array}{l}\text { Disease duration (months) } \\
\text { mean } \pm \text { SD }\end{array}$ & $10.6 \pm 8.5$ & $12.6 \pm 10.4$ & $11.6 \pm 10.5$ & - & n.s. ${ }^{b}$ \\
\hline $\begin{array}{l}\text { Lesion load }(\mathrm{ml}) \\
\text { median (IQR) }\end{array}$ & $2.8(1.4-5.3)$ & $0.9(0.2-2.6)$ & $2.1(0.9-5.3)$ & - & Ci_P vs. Cp_P $: p<0.05^{c}$ \\
\hline EDSS median (IQR) & $3.0(1.5-3.5)$ & $1.5(1.5-2.5)$ & $1.5(1.5-2.5)$ & - & Ci_P vs. Cp_P: $p<0.05^{c}$ \\
\hline $\begin{array}{l}\text { MUSIC cognition score } \\
\text { median (IQR) }\end{array}$ & $13(12-18)$ & $26(23-28)$ & - & - & $\begin{array}{l}\text { Ci_P vs. Cp_P: } \\
p<0.001^{c}\end{array}$ \\
\hline
\end{tabular}

$C i \_P$ cognitively impaired patients, $C p \_P$ cognitively preserved patients, $S D$ standard deviation, $I Q R$ interquartile range, $n$. $s$. not significant

${ }^{\text {a }} \chi^{2}$-square test

$\mathrm{b}$ univariate ANOVA

${ }^{\mathrm{c}}$ Mann-Whitney U-test between Ci_P and Cp_P

Table 2 Fractional anisotropy (FA) in patients with cognitive impairment (Ci_P) compared to cognitively preserved patients $\left(\mathrm{Cp} \_\mathrm{P}\right)$ patients

\begin{tabular}{llll}
\hline FA (mean \pm SD) & Cp_P & Ci_P & $n=14$ \\
\hline Splenium of corpus callosum & $n=81$ & $0.918 \pm 0.05$ & $0.001 *$ \\
Tapetum L & $0.949 \pm 0.02$ & $0.794 \pm 0.10$ & $0.019 *$ \\
Cingulum (hippocampus) R & $0.870 \pm 0.08$ & $0.657 \pm 0.13$ & $0.002 *$ \\
Fornix (cres)/Stria terminalis R & $0.722 \pm 0.08$ & $0.776 \pm 0.08$ & $0.042^{*}$ \\
Posterior corona radiata L & $0.813 \pm 0.05$ & $0.694 \pm 0.05$ & $0.012 *$ \\
Posterior thalamic radiation incl. optic radiation L & $0.731 \pm 0.04$ & $0.799 \pm 0.04$ & 0.050 \\
Cerebral peduncle R & $0.832 \pm 0.04$ & $0.919 \pm 0.04$ & $0.012 *$ \\
Cerebral peduncle L & $0.936 \pm 0.02$ & $0.888 \pm 0.03$ & $<0.001 *$ \\
Medial lemniscus R & $0.913 \pm 0.02$ & $0.858 \pm 0.04$ & $0.013 *$ \\
Medial lemniscus L & $0.878 \pm 0.03$ & $0.808 \pm 0.04$ & $0.024 *$ \\
Cerebellum lobules I-IV L & $0.830 \pm 0.04$ & $0.330 \pm 0.02$ & 0.095 \\
Cerebellum lobule V R & $0.349 \pm 0.03$ & $0.349 \pm 0.03$ & $<0.001 *$ \\
Cerebellum lobule V L & $0.375 \pm 0.02$ & $0.368 \pm 0.03$ & $0.024 *$ \\
Cerebellum lobule VI L & $0.389 \pm 0.02$ & $0.327 \pm 0.03$ & $0.009 *$ \\
\hline
\end{tabular}

Group differences between Ci_P and Cp_P analyzed using a general linear multivariate model controlling for age, sex and EDSS. *: significant after correction for multiple comparisons (Benjamini-Hochberg correction for false discovery rates; FDR at level $\mathrm{q}=0.05$ ). Italics regions which did not survive FDR correction.

$S D$ standard deviation, $F A$ fractional anisotropy, $R$ right hemisphere, $L$ left hemisphere

as fixed factors, and age and EDSS as covariates. We corrected the resulting $p$-values with $p<0.05$ for multiple comparisons by applying a Benjamini-Hochberg correction for false discovery rates (FDR at level $q=0.05$ ).

\section{Results}

\section{Demographic and Clinical Data}

Of the patients $14.7 \%$ (14/95) were classified as cognitively impaired. The demographic results and characteris- tics are summarized in Table 1. In accordance with the inclusion criteria, patients had short disease duration, low grades of brain lesion loads and clinical disability (EDSS). In Ci_P the EDSS and brain lesion load were higher than in $\mathrm{Cp} \_\mathrm{P}$ (both: $p<0.05$ ). Furthermore, the $\mathrm{Ci}$ - $\mathrm{P}$ group contained a higher proportion of male patients and patients at higher age than the $\mathrm{Cp} \_\mathrm{P}$ group. The majority of patients in the Ci_P group showed signs of moderate cognitive impairment, as indicated by the median of the MUSIC cognition score of 13 in this group. 


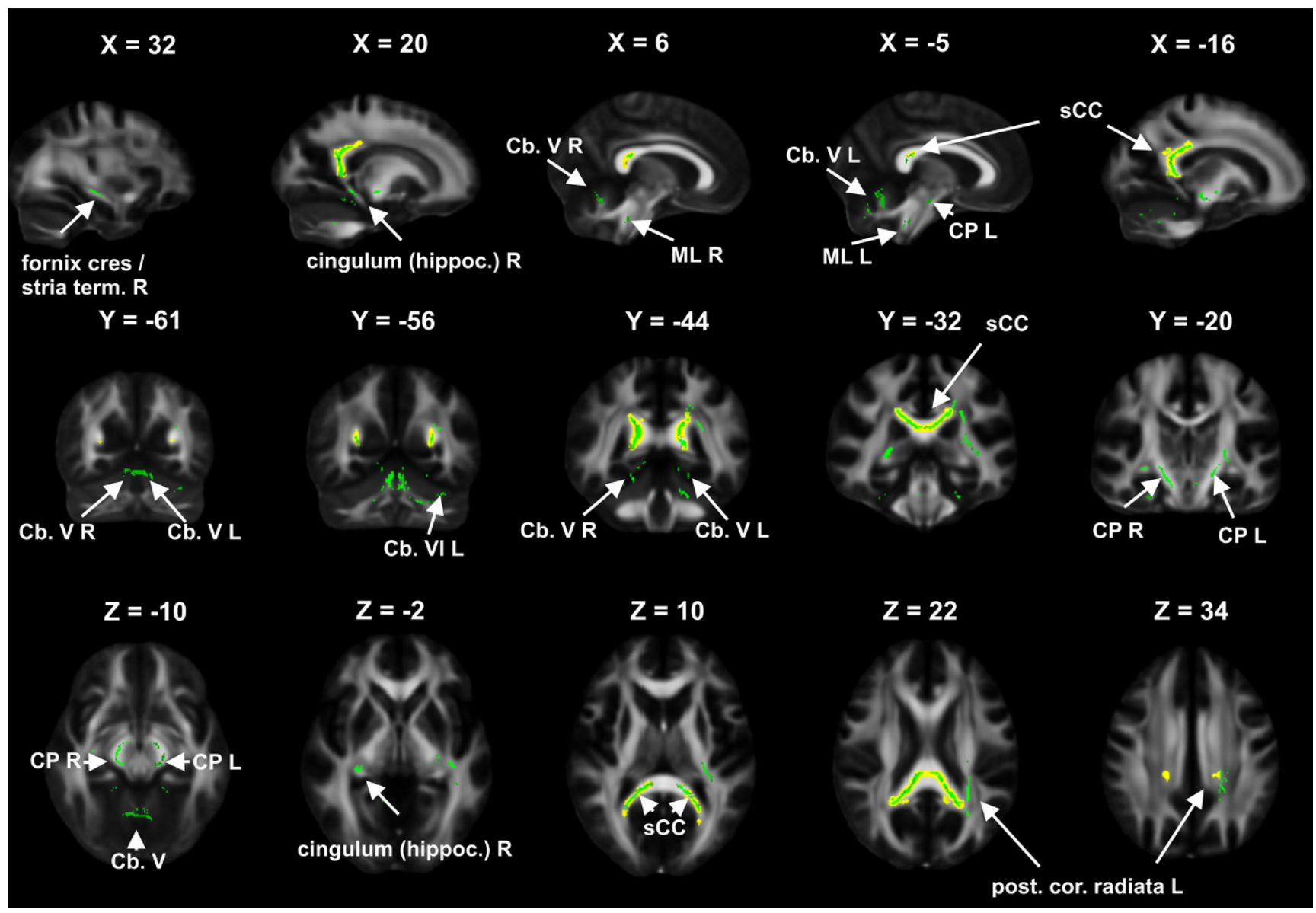

Fig. 2 Differences between cognitively impaired patients $\left(\mathrm{Ci} \_\mathrm{P}\right)$ and cognitively preserved patients $\left(\mathrm{Cp} \_\mathrm{P}\right)$ : the figure demonstrates a voxel-wise group comparison, whereby regions of interest with significant fractional anisotropy (FA) reduction (green overlay) or mean diffusivity (MD) increase (yellow overlay, dilated by 1.5 pixels to improve visibility) in Ci_P compared to Cp_P are shown in sagittal, coronal and axial views on an FA template map in MNI coordinates, X, Y, Z: slices positions in MNI coordinates. The presentation is based on the comparison of the patients and the different groups are not shown here. The green and yellow overlays highlight those clusters of significant differences between patients and healthy controls (based on the preceding tract based spatial statistics analysis [15]) in which stronger involvement was detected in the Ci_P subgroup. FA reduction and MD increase is visible in the splenium of CC. Additional FA reduction is shown in the left hippocampal cingulum, fornix (cres.)/stria terminalis and posterior corona radiata, bihemispheric cerebral peduncle and medial lemnisculus, and in cerebellar lobules $\mathrm{V}$ and VI. $L$ left hemisphere, $R$ right hemisphere, $s C C$ splenium of corpus callosum, post. cor. radiata posterior corona radiata, $C P$ cerebral peduncle, $\mathrm{Cb}$ cerebellum, cingulum (hippoc.) hippocampal cingulum, fornix (cres.)/stria term. fornix (cres.)/stria terminalis, $M L$ medial lemniscus

\section{Diffusion Metrics in Cognitively Impaired Patients Compared to Non-impaired Patients}

Based on TBSS analysis between all patients and HC and atlas-based extraction of quantitative DTI results, we examined associations with $\mathrm{CI}$ for FA, MD and AD in the significant clusters. The analysis included age, sex and EDSS as nuisance variables, since associations between EDSS and FA in different brain structures are well known in MS [14, $28,29]$ and there were between-group differences regarding age and the male/female proportion in our cohort.

The FA showed multiple differences between Ci_P and Cp_P. Details are presented in Table 2 which shows FA for Ci_P and $\mathrm{Cp} \_\mathrm{P}$ in those ROIs where differences between the two groups were significant. Therein, FA was decreased in Ci_P compared to Cp_P in supratentorial, midbrain and pontine and cerebellar WM regions. In detail, we found marked FA reductions $(p \leq 0.002)$ in the splenium of $\mathrm{CC}$, the left cerebral peduncle, the cerebellar lobule (right $\mathrm{V}$ ) and in the right parahippocampal cingulum (cingulum/hippocampus). In addition, FA was reduced in the right fornix (cres)/stria terminalis, left posterior corona radiata and thalamic radiation (including optic radiation), left tapetum, bihemispheric medial lemniscus and cerebellar lobules left I-IV, left V and left VI. The associations with cerebellar lobule left I-IV and left thalamic radiation (incl. optic radiation) did not survive the FDR correction for multiple comparisons. The FA differences in the cerebellar lobule left I-IV and left thalamic radiation (incl. optic radia- 


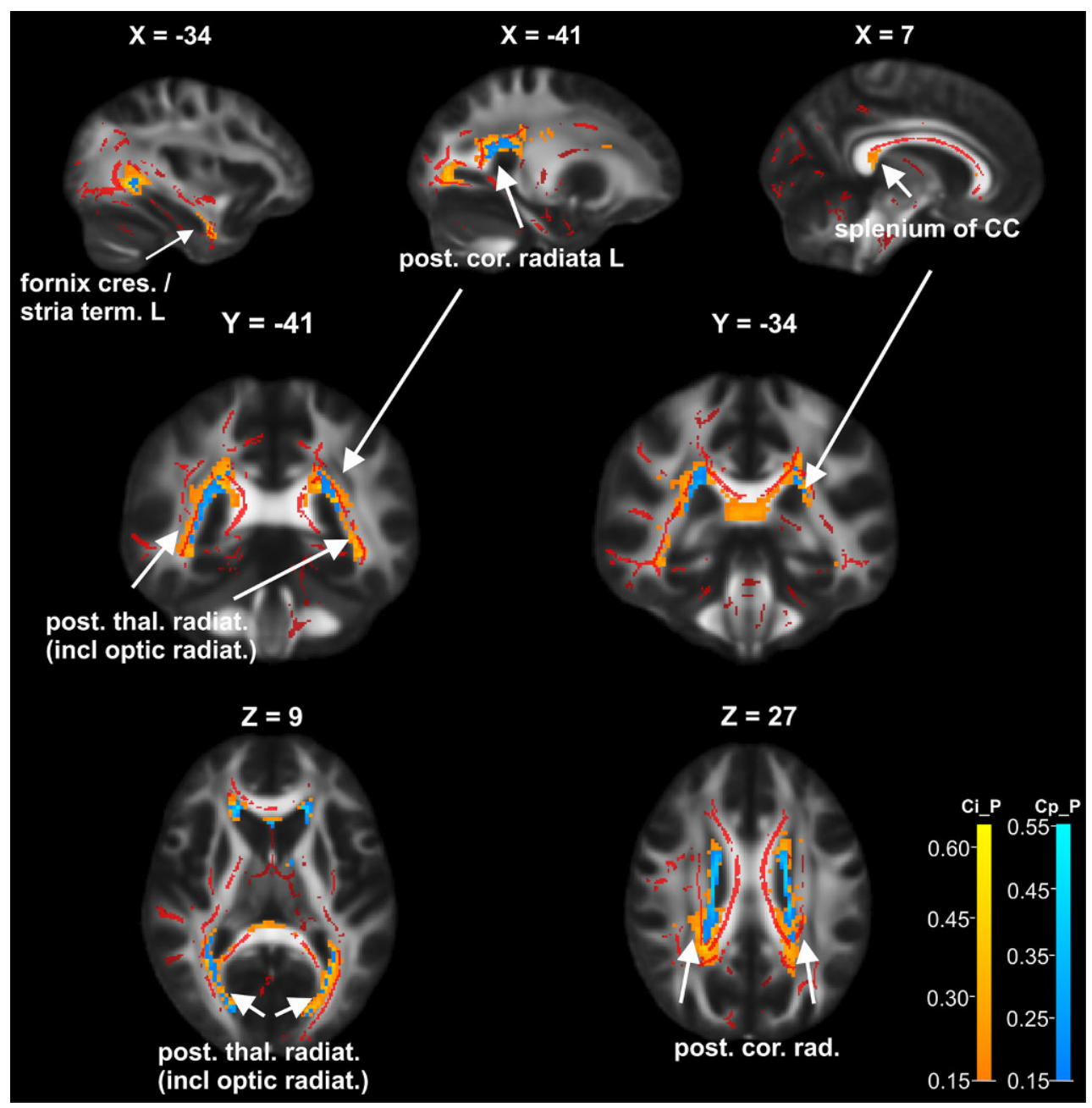

Fig. 3 Lesion probability in cognitively impaired patients $\left(\mathrm{Ci}_{-} \mathrm{P}\right)$ and cognitively preserved patients $\left(\mathrm{Cp} \_\mathrm{P}\right)$ : lesion probability maps of Ci_P (orange/yellow overlay) and Cp_P (light blue overlay) subgroups, and FA clusters of significant differences between patients and healthy controls (red overlay, based on the preceding TBSS analysis [15]) are shown in sagittal, coronal and axial views on an FA template map in MNI coordinates, X, Y, Z: slices positions in MNI coordinates. The lesion probability maps were thresholded at 0.15 , thus voxels in which at least $15 \%$ of patients had FLAIR lesions are displayed. Areas of high lesion probability which were detected bilaterally in the posterior corona radiata and posterior thalamic radiation were found in both, Ci_P and in Cp_P, with larger involvement in Ci_P. Exclusive lesion load detected in the Ci_P group is depicted in the splenium of the corpus callosum and the bilateral fornix (cres)/stria terminalis, respectively temporal WM. $L$ left hemisphere, $R$ right hemisphere, post. cor. rad. posterior corona radiata, ant. cor. rad. anterior corona radiata, sup. cor. rad. superior corona radiata, post. thal. radiat. (incl. optic radiat.) posterior thalamic radiation (including optic radiation), fornix (cres.)/stria term. fornix (cres)/stria terminalis

tion) were not significant after FDR correction for multiple comparisons.

MD was significantly increased in the splenium of CC $(p=0.010)$ in $\mathrm{Ci}-\mathrm{P}\left(43.6 \pm 4.010^{-5} \mathrm{~mm}^{2} / \mathrm{s}\right)$ compared to Cp_P $\left(41.4 \pm 2.210^{-5} \mathrm{~mm}^{2} / \mathrm{s}\right)$. Fig. 2 further illustrates the localization of the ROIs in which significantly altered FA or MD have been detected in Ci_P compared to Cp_P. Only those clusters of significantly altered DTI metrics in patients compared to HC (based on the preceding TBSS analysis) have been highlighted in the figure, in which the Ci_P subgroup was more strongly involved than the $\mathrm{Cp} \_\mathrm{P}$ group.

Regarding AD, for which only a single significant cluster had been detected in the TBSS analysis (right superior corona radiata), there were no significant group differences between Ci_P and Cp_P. For RD we had not detected significant clusters in the TBSS analysis.

\section{Lesion Distribution in Cognitively Impaired Patients and in Non-impaired Patients}

The local distribution of the T2-FLAIR lesions was mainly restricted to periventricular, callosal and temporal regions. Fig. 3 shows the lesion distribution probability maps of Ci_P and Cp_P subgroups and the FA clusters of significant differences between patients and healthy controls (based on the preceding TBSS analysis): lesion probability distributions were similar in both groups with larger involvement 
in Ci_P (orange) compared to Cp_P (light blue) bilaterally in the posterior corona radiata and posterior thalamic radiation, and exclusive lesion load in $\mathrm{Ci}_{-} \mathrm{P}$ in the splenium of the corpus callosum (CC) and the bilateral fornix (cres)/stria terminalis or temporal WM. There was only partial overlap between areas of high lesion probability and clusters on the WM skeleton in which the DTI metrics were significantly altered in Ci_P, namely in the splenium of CC, bilateral posterior corona radiata and left hemispheric fornix (cres)/ stria terminalis.

\section{Discussion}

In the present monocentric analysis, quantitative DTI results representing microstructural WM alterations showed specific differences between patients with CI and cognitively preserved patients at first diagnosis of MS or CIS. While previous studies using conventional MRI measures could not show an association with CI in early MS [8, 9], our study results demonstrate that quantitative DTI seemed to be more sensitive to detect subtle WM alterations related to CI. Compared with Cp_P, FA was reduced in Ci_P in supratentorial and infratentorial WM regions including cerebellar WM regions. Higher MD in Ci_P compared to Cp_P could be detected only in the splenium of the corpus callosum, while AD did not differ between the two groups. Therefore, we focused the discussion on FA differences. We could identify regional clusters of pronounced FA changes between Ci_P and Cp_P. Therefore, anatomical regions mainly related to cognition with significant changes between the patient groups are discussed separately.

Corpus callosum: our results demonstrated a pronounced involvement of the splenium but not of the body of CC in cognitively impaired patients at disease onset in terms of significantly reduced FA (Table 2) and increased MD $(p=0.010)$. This was analogous to earlier studies on patients with long-standing MS, in which associations between microstructural damage in the CC and CI have been shown $[13,30]$. Other studies have reported associations of FA reduction with $\mathrm{CI}$ in the splenium and additionally in the body of CC in established MS [14, 28]. Different fiber types that constitute the $\mathrm{CC}$ may contribute to a varying vulnerability of callosal regions to microstructural damage: while the splenium is mainly composed of densely packed small diameter fibers, large myelinated fibers are predominantly found in the CC body. Thus, the splenium might be more sensitive to microstructural damage than the body of CC and might be particularly involved in early stages of MS [31]. Previous DTI tractography studies on healthy adults have indicated that information processing speed is probably the main mediator between WM microstructural properties and cognitive performance. Explicitly, FA values of the CC splenium were associated with information processing speed $[32,33]$. Accordingly, we hypothesized that in patients with $\mathrm{CI}$ at disease onset, WM integrity changes in the splenium of $\mathrm{CC}$ are relevant for cognitive tasks which reflect information processing speed.

Parahippocampal cingulum and fornix: furthermore, in Ci_P there was significant FA reduction compared to Cp_P in the right hemispheric parahippocampal part of the cingulum and the fornix (cres)/stria terminals (Fig. 3). Damage of the fornix as the major output of the hippocampus can result in severe memory deficits. Thus, microstructural changes in the fornix were related to reduced verbal memory performance in previous studies. MS patients with better memory performance had higher FA in the fornix than patients with memory deficits $[34,35]$. The parahippocampal cingulum contains several hippocampal afferents, and the cingulum as a whole is known to play a critical role in CI because it mediates memory content integration from different parts of the brain [36]. Relations of microstructural changes in the posterior cingulum and fornix reflected by FA reduction and cognitive dysfunction have been shown by Dineen et al. in MS patients with 10-year disease duration [37]. Our results suggest that even in patients at disease onset, CI-related microstructural alterations of fornix and cingulum are detectable. Fornix and cingulum are interrelated structures which are part of the thalamic-hippocampal connections. The interrelation of thalamic atrophy with cognitive performance was shown by Kern et al. who demonstrated that hippocampal-thalamic-prefrontal disruption affected cognitive performance in early MS [38]. These findings were corroborated by a functional connectivity study, which demonstrated reduced connectivity between posterior thalamic nuclei and left supramarginal gyrus, as well as decreased right medial thalamic nuclei connectivity with thalamus and cerebellar areas [39]. In this light, our findings of a pronounced microstructural affection in interrelated thalamic-hippocampal WM regions in cognitively impaired MS patients seem to be conclusive, although we did not investigate thalamic atrophy or brain atrophy in general in the present study.

Cerebellar white matter structures: we additionally detected FA reduction in $\mathrm{Ci}$-P compared to $\mathrm{Cp}$-P in cerebellar structures. Associations between cognition and cerebellar structures have been described in functional MRI studies in the context of corticocerebellar information processing and have also been discussed and corroborated in DTI studies [40, 41]. Especially the cerebellar lobule VI, in which pronounced FA reduction in Ci_P compared to Cp_P was detected in our study, has been suggested to be part of a nonformal cognitive map in the posterior cerebellum contributing to working memory functions and information proceeding speed [40]. Cognitive proceeding speed and working memory capacity, which are equally represented by 
the MUSIC sum score, are two of the most affected cognitive domains in MS [42]. Our study results confirm the relevant alteration of cerebellar WM microstructures (lobule VI left and lobule V) in early MS and CIS patients with CI.

Cognitive impairment in MS is complex and certainly has multifactorial causes, which cannot be fully explained by altered diffusion metrics. Still, in the present patient group with CIS and early MS, the microstructural alterations in Ci_P were mainly located in tracts which are known to be relevant for cognitive performance, reflecting the neural network character of cognitive functions by involvement of parts of the hippocampal-thalamic-prefrontal network and the corticocerebellar loop. Demyelinating lesions leading to impaired WM tract integrity can be a part of the pathophysiological background of the microstructural observations. In this study, we found only partial overlap between areas of cognitively relevant tract locations and areas of high lesion probability. This indicated that normally appearing WM alterations also contributed to cognitive impairment. Still, the subgroup comparison showed specific areas of high lesion probability in the Ci_P group in the CC splenium and in temporal WM intersected by the fornix (cres)/stria terminalis. Thus, the comparison of Ci_P and $\mathrm{Cp} \_\mathrm{P}$ might be influenced by the differences in the subgroup lesion loads and differences in lesion probabilities between the two groups, as patients with early cognitive impairment might have a higher probability for demyelinating lesions in cognitively relevant structures [43].

We acknowledge limitations of our study. We used a global cognitive performance measure for CI representing the most frequently affected cognitive functions in MS. As a result, we could not assign dysfunctions of more specific cognitive domains to regional WM damage. We rather focused on a characterization of microstructural WM damage of Ci_P compared to Cp_P at the earliest stages of MS. Furthermore, although representing the typical features of a very early MS cohort, the number of cognitively impaired patients was relatively small compared to the cognitively preserved patients. Thus, in future investigations larger numbers of patients, possibly in multicentric analyses, should be included. Furthermore, CI cannot exclusively be attributed to structural impairment of brain WM, due to the known evidence of the associations of cortical lesions and cortical atrophy with CI in RRMS [44], and moreover in progressive MS and patients with longer disease duration [45]. Although contributions of GM pathology to CI have also been reported for CIS and early MS [46], these effects have not been taken into account in this study and must therefore be mentioned as a limitation. A further limitation might be introduced by the definition of the ROIs in which the DTI metrics have been extracted: since the analyses were based on clusters of significant differences between patients and healthy controls gained from the preceding study, and the entire patient group was dominated by Cp_P, regions with specific involvement in $\mathrm{Ci} \_\mathrm{P}$ might have been underestimated or missed by the chosen methodology. Still, the number of those potentially missing regions is probably small since our analyses included regions of nearly all tracts of the JHU-ICBM WM label atlas and most large tracts of the cerebellar atlas. Future studies, including larger numbers of patients should directly investigate the voxelwise comparison between $\mathrm{Ci}$ _P and Cp_P. In addition, we used a 32 gradient direction DTI sequence with limited spatial resolution of $2.5 \mathrm{~mm}$, therefore higher resolution and number of gradient directions would be desirably in future studies. Moreover, future studies should investigate these effects using advanced multi-shell diffusion protocols (e.g. NODDI [47]), since these models measure complementary WM properties and might be more closely related to cognitive performance than DTI $[48,49]$.

\section{Conclusion}

Referring to our hypotheses, we were able to confirm that microstructural WM alterations were more pronounced in known cognitively relevant tracts of cognitively impaired patients, even in the earliest stages of MS disease. Particularly, the splenium of corpus callosum, fornix, parahippocampal cingulum and cerebellar WM tracts seemed to be relevant for CI. Considering the impact of cognitive impairment on occupational disability and restrictions in quality of life for patients with MS these pronounced alterations of WM integrity in $\mathrm{Ci}_{-} \mathrm{P}$ at disease onset underline the relevance of early treatment initiation.

Supplementary Information The online version of this article (https:// doi.org/10.1007/s00062-021-01010-8) contains supplementary material, which is available to authorized users.

Funding Part of this work was supported by the German Federal Ministry for Education and Research, BMBF, German Competence Network Multiple Sclerosis KKNMS (grant 01GI1601I). EG received financial support by the Deutsche Forschungsgemeinschaft (DFG) grant number GE 2777/2-1 and project number: 316803389-SFB 1280 project $\mathrm{A} 03$.

Author Contribution All authors whose names appear on the submission: 1) made substantial contributions to the conception or design of the work or the acquisition, analysis, or interpretation of data, 2) drafted the work or revised it critically for important intellectual content, 3) approved the version to be published and 4) agree to be accountable for all aspects of the work in ensuring that questions related to the accuracy or integrity of any part of the work are appropriately investigated and resolved. Personalized authorship contributions: Ruth Schneider: conceptualization, data curation, formal analysis, investigation, methodology,; project administration, visualization, writing original draft, writing review and editing. Britta Matusche: data curation, formal analysis, visualization, writing review and editing. $\mathrm{Er}$ han Genç: conceptualization, data curation, formal analysis, method- 
ology, software, writing review and editing. Ralf Gold: conceptualization, funding acquisition, resources, supervision, writing review and editing. Barbara Bellenberg: conceptualization, data curation, formal analysis, investigation, methodology, project administration, visualization, writing original draft, writing review and editing. Carsten Lukas: conceptualization, funding acquisition, investigation, methodology, resources, supervision, writing review and editing.

Funding Open Access funding enabled and organized by Projekt DEAL.

\section{Declarations}

Conflict of interest R. Schneider has received consulting and speakers honoraria from Biogen Idec GmBH and Roche Pharma AG \& has received research scientific grant support from Novartis Pharma. R. Gold has received compensation for serving as a consultant or speaker from Bayer HealthCare, Biogen Idec, Merck Serono, Novartis, and Teva Neuroscience; he, or the institution he works for, has received research support from Bayer HealthCare, Biogen Idec, Merck Serono, Novartis and Teva Neuroscience; he has also received honoraria as a Journal Editor from SAGE and Thieme Verlag. B. Bellenberg received financial support by the German Federal Ministry for Education and Research, BMBF, German Competence Network Multiple Sclerosis (KKNMS), grant no.01GI1601I. C. Lukas received a research grant by the German Federal Ministry for Education and Research, BMBF, German Competence Network Multiple Sclerosis (KKNMS), grant no.01GI1601I, has received consulting and speaker's honoraria from Biogen Idec, Bayer Schering, Daiichi Sanykyo, Merck Serono, Novartis, Sanofi, Genzyme and TEVA. B. Matusche and E. Genç declare that they have no competing interests.

Ethical standards The study was approved by the local ethics committee of the Ruhr University Bochum (Approval No. 3714-10). The study was performed in accordance with the ethical standards laid down in the 1964 Declaration of Helsinki and its later amendments. Consent to participate: all participants gave written informed consent for participation in the study, which includes dissemination of the results.

Open Access This article is licensed under a Creative Commons Attribution 4.0 International License, which permits use, sharing, adaptation, distribution and reproduction in any medium or format, as long as you give appropriate credit to the original author(s) and the source, provide a link to the Creative Commons licence, and indicate if changes were made. The images or other third party material in this article are included in the article's Creative Commons licence, unless indicated otherwise in a credit line to the material. If material is not included in the article's Creative Commons licence and your intended use is not permitted by statutory regulation or exceeds the permitted use, you will need to obtain permission directly from the copyright holder. To view a copy of this licence, visit http://creativecommons.org/licenses/by/4. $0 \%$.

\section{References}

1. DeLuca GC, Yates RL, Beale H, Morrow SA. Cognitive impairment in multiple sclerosis: clinical, radiologic and pathologic insights. Brain Pathol. 2015;25:79-98.

2. Amato MP, Zipoli V, Portaccio E. Multiple sclerosis-related cognitive changes: a review of cross-sectional and longitudinal studies. J Neurol Sci. 2006;245:41-6.

3. Hynčicová E, Vyhnálek M, Kalina A, Martinkovič L, Nikolai T, Lisý J, Hort J, Meluzínová E, Laczó J. Cognitive impairment and structural brain changes in patients with clinically isolated syndrome at high risk for multiple sclerosis. J Neurol. 2017;264:482-93.

4. Uher T, Blahova-Dusankova J, Horakova D, Bergsland N, Tyblova M, Benedict RH, Kalincik T, Ramasamy DP, Seidl Z, Hagermeier J, Vaneckova M, Krasensky J, Havrdova E, Zivadinov R. Longitudinal MRI and neuropsychological assessment of patients with clinically isolated syndrome. J Neurol. 2014;261:1735-44.

5. Macías Islas MÁ, Ciampi E. Assessment and Impact of Cognitive Impairment in Multiple Sclerosis: An Overview. Biomedicines. 2019;7:22.

6. Ozakbas S, Turkoglu R, Tamam Y, Terzi M, Taskapilioglu O, Yucesan C, Baser HL, Gencer M, Akil E, Sen S, Turan OF, Sorgun MH, Yigit P, Turkes N. Prevalence of and risk factors for cognitive impairment in patients with relapsing-remitting multiple sclerosis: Multi-center, controlled trial. Mult Scler Relat Disord. 2018;22:70-6.

7. Paul F. Pathology and MRI: exploring cognitive impairment in MS. Acta Neurol Scand. 2016;134 Suppl 200:24-33.

8. Uher T, Krasensky J, Sobisek L, Blahova Dusankova J, Seidl Z, Kubala Havrdova E, Sormani MP, Horakova D, Kalincik T, Vaneckova M. Cognitive clinico-radiological paradox in early stages of multiple sclerosis. Ann Clin Transl Neurol. 2017;5:81-91.

9. Johnen A, Bürkner PC, Landmeyer NC, Ambrosius B, Calabrese P, Motte J, Hessler N, Antony G, König IR, Klotz L, Hoshi MM, Aly L, Groppa S, Luessi F, Paul F, Tackenberg B, Bergh FT, Kümpfel T, Tumani H, Stangel M, Weber F, Bayas A, Wildemann B, Heesen C, Zettl UK, Zipp F, Hemmer B, Meuth SG, Gold R, Wiendl H, Salmen A; German Competence Network Multiple Sclerosis (KKNMS). Can we predict cognitive decline after initial diagnosis of multiple sclerosis? Results from the German National early MS cohort (KKNMS). J Neurol. 2019;266:386-97.

10. Engl C, Tiemann L, Grahl S, Bussas M, Schmidt P, Pongratz V, Berthele A, Beer A, Gaser C, Kirschke JS, Zimmer C, Hemmer B, Mühlau M. Cognitive impairment in early MS: contribution of white matter lesions, deep grey matter atrophy, and cortical atrophy. J Neurol. 2020;267:2307-18.

11. Aung WY, Mar S, Benzinger TL. Diffusion tensor MRI as a biomarker in axonal and myelin damage. Imaging Med. 2013;5: 427-40.

12. Rovira A, Auger C, Alonso J. Magnetic resonance monitoring of lesion evolution in multiple sclerosis. Ther Adv Neurol Disord. 2013;6:298-310.

13. Hulst HE, Steenwijk MD, Versteeg A, Pouwels PJ, Vrenken H, Uitdehaag BM, Polman CH, Geurts JJ, Barkhof F. Cognitive impairment in MS: impact of white matter integrity, gray matter volume, and lesions. Neurology. 2013;80:1025-32.

14. Huang J, Liu Y, Zhao T, Shu N, Duan Y, Ren Z, Sun Z, Liu Z, Chen H, Dong H, Li K. White matter microstructural alterations in clinically isolated syndrome and multiple sclerosis. J Clin Neurosci. 2018;53:27-33.

15. Schneider R, Genç E, Ahlborn C, Gold R, Lukas C, Bellenberg B. Temporal Dynamics of Diffusion Metrics in Early Multiple Sclerosis and Clinically Isolated Syndrome: A 2-Year Follow-Up TractBased Spatial Statistics Study. Front Neurol. 2019;10:1165.

16. Polman CH, Reingold SC, Edan G, Filippi M, Hartung HP, Kappos L, Lublin FD, Metz LM, McFarland HF, O'Connor PW, SandbergWollheim M, Thompson AJ, Weinshenker BG, Wolinsky JS. Diagnostic criteria for multiple sclerosis: 2005 revisions to the "McDonald Criteria”. Ann Neurol. 2005;58:840-6.

17. Calabrese P, Kalbe E, Kessler J. Ein neuropsychologisches Screening zur Erfassung kognitiver Störungen bei MS-Patienten - Das Multiple Sklerose Inventarium Cognition (MUSIC). psychoneuro. 2004;30:384-8.

18. Espinosa A, Alegret M, Valero S, Vinyes-Junqué G, Hernández I, Mauleón A, Rosende-Roca M, Ruiz A, López O, Tárraga L, Boada M. A longitudinal follow-up of 550 mild cognitive impairment pa- 
tients: evidence for large conversion to dementia rates and detection of major risk factors involved. J Alzheimers Dis. 2013;34:769-80.

19. Kalbe E, Calabrese P, Fengler S, Kessler J. DemTect, PANDA, EASY, and MUSIC: cognitive screening tools with age correction and weighting of subtests according to their sensitivity and specificity. J Alzheimers Dis. 2013;34:813-34.

20. von Bismarck O, Dankowski T, Ambrosius B, Hessler N, Antony G, Ziegler A, Hoshi MM, Aly L, Luessi F, Groppa S, Klotz L, Meuth SG, Tackenberg B, Stoppe M, Then Bergh F, Tumani H, Kümpfel T, Stangel M, Heesen C, Wildemann B, Paul F, Bayas A, Warnke C, Weber F, Linker RA, Ziemann U, Zettl UK, Zipp F, Wiendl H, Hemmer B, Gold R, Salmen A. Treatment choices and neuropsychological symptoms of a large cohort of early MS. Neurol Neuroimmunol Neuroinflamm. 2018;5:e446.

21. Kurtzke JF. Rating neurologic impairment in multiple sclerosis: an expanded disability status scale (EDSS). Neurology. 1983;33:1444-52.

22. Smith SM, Jenkinson M, Johansen-Berg H, Rueckert D, Nichols TE, Mackay CE, Watkins KE, Ciccarelli O, Cader MZ, Matthews PM, Behrens TE. Tract-based spatial statistics: voxelwise analysis of multi-subject diffusion data. Neuroimage. 2006;31:1487-505.

23. Smith SM, Jenkinson M, Woolrich MW, Beckmann CF, Behrens TE, Johansen-Berg H, Bannister PR, De Luca M, Drobnjak I, Flitney DE, Niazy RK, Saunders J, Vickers J, Zhang Y, De Stefano N, Brady JM, Matthews PM. Advances in functional and structural MR image analysis and implementation as FSL. Neuroimage. 2004;23 Suppl 1:S208-19.

24. Winkler AM, Ridgway GR, Webster MA, Smith SM, Nichols TE. Permutation inference for the general linear model. Neuroimage. 2014;92:381-97.

25. Mori S, Oishi K, Jiang H, Jiang L, Li X, Akhter K, Hua K, Faria AV, Mahmood A, Woods R, Toga AW, Pike GB, Neto PR, Evans A, Zhang J, Huang H, Miller MI, van Zijl P, Mazziotta J. Stereotaxic white matter atlas based on diffusion tensor imaging in an ICBM template. Neuroimage. 2008;40:570-82.

26. Diedrichsen J, Balsters JH, Flavell J, Cussans E, Ramnani N. A probabilistic MR atlas of the human cerebellum. Neuroimage. 2009;46:39-46.

27. Schmidt P, Gaser C, Arsic M, Buck D, Förschler A, Berthele A, Hoshi M, Ilg R, Schmid VJ, Zimmer C, Hemmer B, Mühlau M. An automated tool for detection of FLAIR-hyperintense white-matter lesions in Multiple Sclerosis. Neuroimage. 2012;59:3774-83.

28. Caligiuri ME, Barone S, Cherubini A, Augimeri A, Chiriaco C, Trotta M, Granata A, Filippelli E, Perrotta P, Valentino P, Quattrone A. The relationship between regional microstructural abnormalities of the corpus callosum and physical and cognitive disability in relapsing-remitting multiple sclerosis. Neuroimage Clin. 2014;7:28-33.

29. Welton T, Kent D, Constantinescu CS, Auer DP, Dineen RA. Functionally relevant white matter degradation in multiple sclerosis: a tract-based spatial meta-analysis. Radiology. 2015;275:89-96.

30. Huang SY, Fan Q, Machado N, Eloyan A, Bireley JD, Russo AW, Tobyne SM, Patel KR, Brewer K, Rapaport SF, Nummenmaa A, Witzel T, Sherman JC, Wald LL, Klawiter EC. Corpus callosum axon diameter relates to cognitive impairment in multiple sclerosis. Ann Clin Transl Neurol. 2019;6:882-92.

31. Aboitiz F, Scheibel AB, Fisher RS, Zaidel E. Fiber composition of the human corpus callosum. Brain Res. 1992;598:143-53.

32. Zarei M, Johansen-Berg H, Smith S, Ciccarelli O, Thompson AJ, Matthews PM. Functional anatomy of interhemispheric cortical connections in the human brain. J Anat. 2006;209:311-20.

33. Ferrer E, Whitaker KJ, Steele JS, Green CT, Wendelken C, Bunge SA. White matter maturation supports the development of reasoning ability through its influence on processing speed. Dev Sci. 2013;16:941-51.
34. Kern KC, Ekstrom AD, Suthana NA, Giesser BS, Montag M, Arshanapalli A, Bookheimer SY, Sicotte NL. Fornix damage limits verbal memory functional compensation in multiple sclerosis. Neuroimage. 2012;59:2932-40.

35. Gaffan D, Gaffan EA. Amnesia in man following transection of the fornix. A review. Brain. 1991;114:2611-8.

36. Metzler-Baddeley C, Jones DK, Steventon J, Westacott L, Aggleton JP, O'Sullivan MJ. Cingulum microstructure predicts cognitive control in older age and mild cognitive impairment. J Neurosci. 2012;32:17612-9.

37. Dineen RA, Vilisaar J, Hlinka J, Bradshaw CM, Morgan PS, Constantinescu CS, Auer DP. Disconnection as a mechanism for cognitive dysfunction in multiple sclerosis. Brain. 2009;132:239-49.

38. Kern KC, Gold SM, Lee B, Montag M, Horsfall J, O'Connor MF, Sicotte NL. Thalamic-hippocampal-prefrontal disruption in relapsing-remitting multiple sclerosis. Neuroimage Clin. 2014;8:440-7.

39. Lin F, Zivadinov R, Hagemeier J, Weinstock-Guttman B, Vaughn C, Gandhi S, Jakimovski D, Hulst HE, Benedict RH, Bergsland N, Fuchs T, Dwyer MG. Altered nuclei-specific thalamic functional connectivity patterns in multiple sclerosis and their associations with fatigue and cognition. Mult Scler. 2019;25:1243-54.

40. Moroso A, Ruet A, Lamargue-Hamel D, Munsch F, Deloire M, Coupé P, Charré-Morin J, Saubusse A, Ouallet JC, Planche V, Tourdias T, Dousset V, Brochet B. Microstructural analyses of the posterior cerebellar lobules in relapsing-onset multiple sclerosis and their implication in cognitive impairment. PLoS One. 2017;12:e0182479.

41. Hayter AL, Langdon DW, Ramnani N. Cerebellar contributions to working memory. Neuroimage. 2007;36:943-54.

42. Chiaravalloti ND, DeLuca J. Cognitive impairment in multiple sclerosis. Lancet Neurol. 2008;7:1139-51.

43. Rossi F, Giorgio A, Battaglini M, Stromillo ML, Portaccio E, Goretti B, Federico A, Hakiki B, Amato MP, De Stefano N. Relevance of brain lesion location to cognition in relapsing multiple sclerosis. PLoS One. 2012;7:e44826.

44. Calabrese M, Agosta F, Rinaldi F, Mattisi I, Grossi P, Favaretto A, Atzori M, Bernardi V, Barachino L, Rinaldi L, Perini P, Gallo P, Filippi M. Cortical lesions and atrophy associated with cognitive impairment in relapsing-remitting multiple sclerosis. Arch Neurol. 2009;66:1144-50.

45. Calabrese M, Reynolds R, Magliozzi R, Castellaro M, Morra A, Scalfari A, Farina G, Romualdi C, Gajofatto A, Pitteri M, Benedetti MD, Monaco S. Regional Distribution and Evolution of Gray Matter Damage in Different Populations of Multiple Sclerosis Patients. PLoS One. 2015;10:e0135428.

46. Dalton CM, Chard DT, Davies GR, Miszkiel KA, Altmann DR, Fernando K, Plant GT, Thompson AJ, Miller DH. Early development of multiple sclerosis is associated with progressive grey matter atrophy in patients presenting with clinically isolated syndromes. Brain. 2004;127:1101-7.

47. Zhang H, Schneider T, Wheeler-Kingshott CA, Alexander DC. NODDI: practical in vivo neurite orientation dispersion and density imaging of the human brain. Neuroimage. 2012;61:1000-16.

48. Friedrich P, Fraenz C, Schlüter C, Ocklenburg S, Mädler B, Güntürkün O, Genç E. The Relationship Between Axon Density, Myelination, and Fractional Anisotropy in the Human Corpus Callosum. Cereb Cortex. 2020;30:2042-56.

49. Genç E, Fraenz C, Schlüter C, Friedrich P, Hossiep R, Voelkle MC, Ling JM, Güntürkün O, Jung RE. Diffusion markers of dendritic density and arborization in gray matter predict differences in intelligence. Nat Commun. 2018;9:1905. 